\section{LA ECONOMÍA DEL MAR. LOS RECURSOS MARINOS DE LA PLATAFORMA CONTINENTAL AMPLIADA}

\author{
THE ECONOMY OF THE SEA. MARINE RESOURCES \\ OF EXTENDED CONTINENTAL SHELF
}

Micaela A. Spinelli | Valeria Tomasini | Enrique Dentice

\section{RESUMEN}

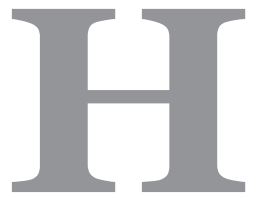
ay una Argentina que se ve y otra oculta bajo el mar, con un enorme potencial de recursos y que es la prolongación natural de nuestro territorio. Gracias al trabajo científico, técnico y jurídico realizado durante los últimos 20 años esa Argentina se pudo conocer mejor, permitiendo demarcar el nuevo límite exterior de la Plataforma Continental Argentina. La propuesta la presentó el país en 2009 ante la Comisión de Límites de la Plataforma Continental y fue estudiado desde 2012 por una comisión de expertos que analizó los dictámenes de geólogos, hidrólogos y diplomáticos argentinos sobre algo que parecía sin importancia: ¿cuánto mide la plataforma submarina? Ese trabajo se acumuló en decenas de documentos, mapas y demás testimonios durante varios años que, finalmente, llevaron a la Argentina a ampliar su plataforma continental de las 200 millas a las 350 millas incorporando así, más de un $35 \%$ más que la superficie original. De esta manera, la plataforma continental argentina tiene una superficie de más de $6.500 .000 \mathrm{~km} 2$, casi el doble que la superficie emergida de la Argentina. Esta valiosa ampliación, además de reafirmar nuestros derechos sobre minerales, hidrocarburos y especies ictícolas, se traduce en implicancias por demás favorables para los intereses argentinos en temas como Malvinas, Islas del Atlántico Sur y Antártida. Se abordará en el presente trabajo el detalle de esa exhaustiva investigación, como así también el impacto económico y los recursos implicados en la misma.

Palabras clave: plataforma continental, recursos marinos, potencialidad, medio ambiente y control de los recursos.

\section{ABSTRACT}

There is a visible Argentina and another hidden under the sea. The latter is the natural extension of the mailand and has an enourmous potential in
Micaela A. Spinelli mspinelli@unsam.edu.ar

Valeria Tomasini valeria.tomasini@unsam.edu.ar

Enrique Dentice edentice@unsam.edu.ar

Centro de Investigación y Medición Económica - CIME.

Universidad Nacional de San Martín

ARGENTINA

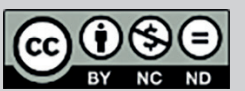

https://creativecommons.org/licenses/by-nc-nd/4.0/ Revista de la Facultad de Ciencias Económicas ISSN 1668-6357 (formato impreso) ISSN 1668-6365 (formato digital) por Facultad de Ciencias Económicas Universidad Nacional del Nordeste (UNNE) Argentina se distribuye bajo una Licencia Creative Commons Atribución - No Comercial - Sin Obra Derivada 4.0 Internacional. 
terms of resources. Thanks to the scientific, technical and legal work carried out during the last 20 years, we have a better picture of this hidden Argentina, allowing a new delimitation of the Continental Platform. Argentina presented a proposal to enforce these new limits to the Commission for the Limits of the Continental Shelf in 2009, and was studied since 2012 by a group of experts that analyzed the opinions of geologists, hydrologists and diplomats from Argentina on a seemingly unimportant issue: what is the size of underwater platform? This work was accumulated on severals documents, maps and other testimonies for several years, until Argentina was able to expand its continental platform from 200 miles to 350 miles, expanding the original surface by $35 \%$. The Argentine continental shelf has now a surface area of more than $6,500,000 \mathrm{~km} 2$, almost twice as large as its mainland surface. This valuable expansion, not only reaffirms our rights over minerals, hydrocarbons and fishes, but it also has strategic implications related to The Malvinas, The South Atlantic Islands and The Antarctica. This work addresses the details of this exhaustive investigation, as well as the related economic impacts.

Keywords: continental shelf, marine resources, potentiality, environment and control of resources.

\section{OBJETIVOS}

- Analizar el impacto de la ampliación de la plataforma continental argentina a los efectos de definir los potenciales recursos en materia de hidrocarburos, gas, y especies sedentarias.

- Establecer la necesidad de controlar los recursos como su preservación y su impacto en el medio ambiente.

- Estudiar el nuevo horizonte de desafíos para el control de la zona ampliada.

- Establecer un posible incremento del comercio exterior argentino en peces, mariscos y otros moluscos.

- Verificar el accionar presente y los medios a requerir para el control efectivo de la plataforma continental. 


\section{ACTIVIDADES Y METODOLOGÍA}

- Análisis de la información de la ONU-COPLA- respecto a la zona ampliada.

- Búsqueda de las recomendaciones de los organismos internacionales (CONVEMAR y COPLA).

- Analizar los resultados de la investigación realizadas en el proyecto Pampa Azul.

- Búsqueda de estadísticas de Pesca, especies y tipos de pesca en la economía ribereña de las provincias, para contrastarla con los respectivos PBG.

- Información de carácter relevante en cuanto a explotación hidrocarburífera, gasífera y pesquera, en términos de cuencas, caladeros y potencialidades y reservas.

\section{ANTECEDENTES}

El océano Atlántico comenzó su formación hace 150 millones de años, cuando se inició la separación de América del Sur y África. Los procesos endógenos, que actuaron durante aquella separación, también fueron los responsables de la formación de las cuencas oceánicas y dejaron su impronta a través de una topografía irregular que, a lo largo del tiempo geológico, fue suavizada por los aportes de sedimentos provenientes del continente. Estos materiales se distribuyeron a lo largo de las costas formando la plataforma continental, que ocupa el 7,5\% de los fondos oceánicos del mundo.

Se trata de superficies suavemente inclinadas, pero que, en el caso particular de nuestro país, tiene un ancho considerable. Un promedio de $560 \mathrm{~km}$, que a la altura del golfo San Jorge es de $850 \mathrm{~km}$ y cuya mayor extensión se desarrolla a partir del paralelo de $49^{\circ}$.

La plataforma continental es la prolongación sumergida de la masa terrestre de un estado hasta borde exterior del margen continental, o hasta las 200 millas náuticas (370 km) contadas desde la línea de bases si el borde exterior del margen continental no llega hasta esa distancia, independientemente de las condiciones geológicas o geomorfológicas de esa extensión o de que se extienda o no hasta ese punto la plataforma continental geológica. Sobre la plataforma continental el estado ribereño ${ }^{1}$ ejerce derechos exclusivos de soberanía para la exploración y explotación de los recursos naturales allí existentes ${ }^{2}$. Los fondos oceánicos que queden más allá de los límites que fijen los estados están bajo la jurisdicción de la Autoridad Internacional de los Fondos Marinos y son considerados para beneficio de toda la humanidad. El fondo oceánico profundo con sus crestas oceánicas y su subsuelo queda fuera de la jurisdicción de los estados.

\footnotetext{
${ }^{1}$ Es la soberanía ejercida por un Estado (que tiene costa marina) más allá de sus límites terrestres y de las aguas interiores, extendiéndose al mar. Asímismo, el Estado en ejercicio de su soberanía podrá dictar leyes y reglamentos referidos a esta zona. ${ }^{2}$ Artículo 77 de la Convemar.
} 
Argentina ratificó la convención el 12 de enero de 1995, entrando en vigor el 31 de diciembre de 1995 .

Para establecer hasta dónde se extiende el borde exterior del margen continental y, por lo tanto, establecer el límite exterior de la plataforma continental más allá de las 200 millas náuticas, se utilizan dos criterios, definidos en el artículo 74 inciso 4 de la Convemar:

1) Una línea trazada, de conformidad con el artículo 76 inciso 7 , en relación con los puntos fijos más alejados en cada uno de los cuales el espesor de las rocas sedimentarias sea por lo menos el 1\% de la distancia más corta entre ese punto y el pie del talud continental.

2) Una línea trazada, de conformidad con el artículo 76 inciso 7 , en relación con puntos fijos situados a no más de 60 millas náuticas del pie del talud continental.

El artículo 76 inciso 5 establece además dos restricciones. Los puntos fijos que constituyan el límite exterior de la plataforma continental, trazados de acuerdo con los criterios anteriormente mencionados, no deberán exceder de 350 millas náuticas $(650 \mathrm{~km})$ o de 100 millas náuticas $(190 \mathrm{~km}$ ) desde la isóbata de 2500 metros (la línea que conecta la profundidad de 2500 metros). Los puntos deben unirse mediante líneas rectas, cuya longitud no debe exceder de 60 millas náuticas $(111 \mathrm{~km})$.

\section{PROCEDIMIENTO PARA LA SOLICITACIÓN DE LA EXTENSIÓN}

La posibilidad de que los estados ribereños extiendan su límite exterior de la plataforma continental más allá de las 200 millas náuticas ha sido contemplada por la Convemar para ciertos casos especificados en su artículo 76.1 Los estados interesados en realizar esa extensión deben realizar una presentación fundamentada a la Comisión de Límites de la Plataforma Continental (CLCS), la cual debe evaluar y formular posteriormente recomendaciones para la fijación del límite exterior de la plataforma continental de dicho estado.

Los límites de la plataforma continental no afectan la condición jurídica de las aguas suprayacentes ni a la del espacio aéreo situado sobre tales aguas, de acuerdo con el artículo 78 inciso 1. 

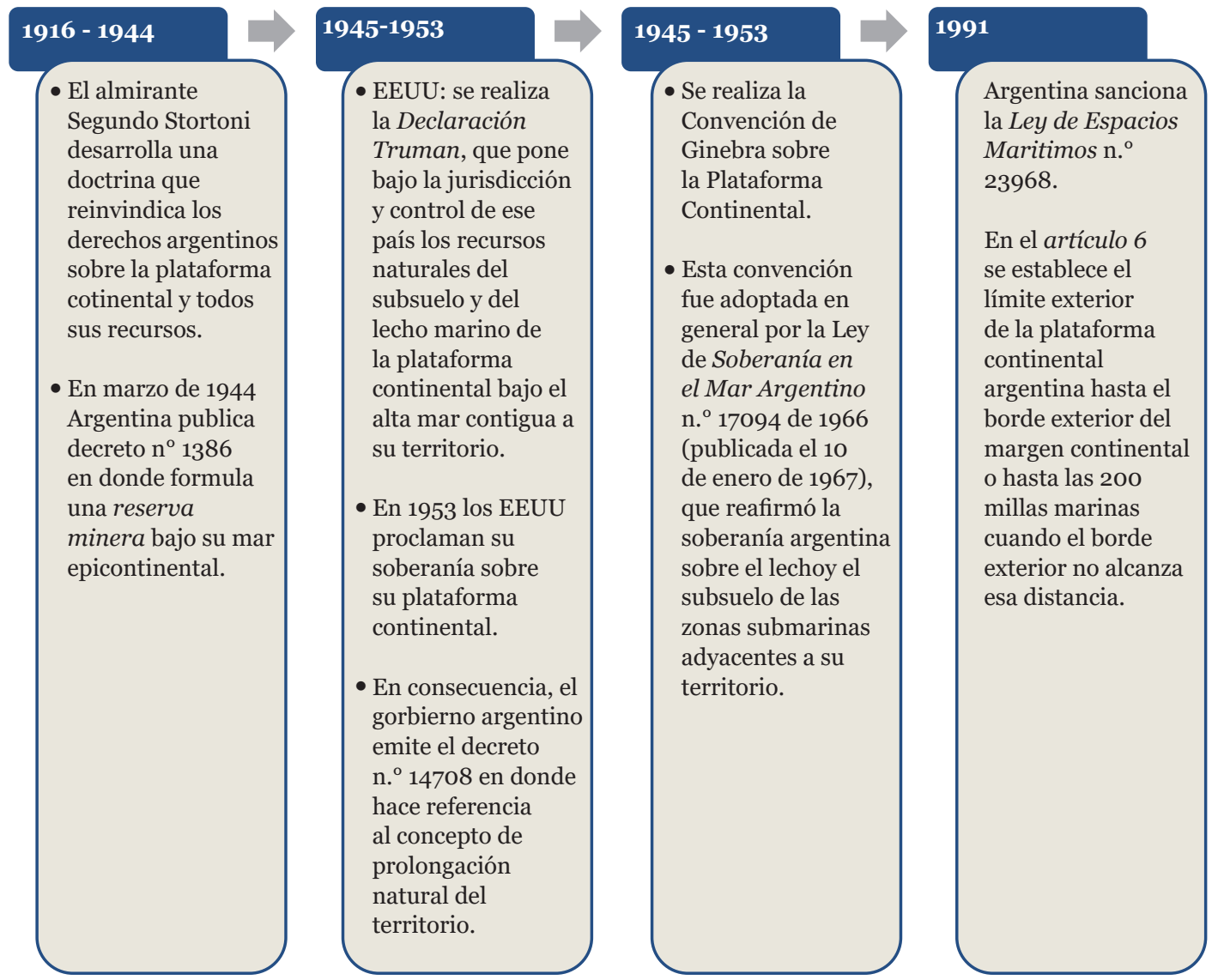

La plataforma continental sobre la cual ejerce soberanía la Nación Argentina, comprende el lecho y el subsuelo de las áreas submarinas que se extienden más allá de su mar territorial y a todo lo largo de la prolongación natural de su territorio hasta el borde exterior del margen continental, o bien hasta una distancia de doscientas (200) millas náuticas medidas a partir de las líneas de base que se establecen en el art. $1^{0}$ de la ley $n^{\circ} 23968$, en los casos en que el borde exterior no llegue a esa distancia. ${ }^{3}$ 


\section{PRESENTACIÓN ARGENTINA}

\section{AGENTES INTERVINIENTES}

\section{CONVENCIÓN DE LAS NACIONES UNIDAS SOBRE EL DERECHO DEL MAR}

La Convención de las Naciones Unidas sobre el Derecho del Mar -de ahora en adelante CONVEMAR- es un tratado multilateral entre 168 países que limitan su territorio marítimo y regula todas las actividades humanas en los océanos y en el mar territorial.

Se caracteriza por confirmar el derecho internacional del mar vigente, al incorporar muchos aspectos de las Convenciones de Ginebra de 1958 y, además, por desarrollar progresivamente el derecho internacional del mar, al establecer nuevos institutos en la materia, como la zona económica exclusiva. Tiene aplicación preferente, entre sus miembros, frente a las Convenciones de Ginebra de 1958; no obstante, las Convenciones de Ginebra siguen teniendo vigencia entre los Estados partes que no han adherido a esta Convención.

El tratado fue aprobado, tras nueve años de trabajo, el 30 de abril de 1982 en Nueva York (Estados Unidos) y abierta a su firma por parte de los Estados, el 10 de diciembre de 1982, en Bahía Montego (Jamaica), en la $182 .^{\circ}$ sesión plenaria de la III Conferencia de las Naciones Unidas sobre el Derecho del Mar. Entró en vigor el 16 de noviembre de 1994.

La Convención del Derecho del Mar consta de un preámbulo, 17 partes y 9 anexos. Entre otros, cubre los siguientes temas de derecho del mar: límites de las zonas marítimas; zona económica exclusiva; plataforma continental y alta mar; derechos de navegación y estrechos para la navegación internacional; estados archipelágicos; paz y la seguridad en los océanos y los mares; conservación y gestión de los recursos marinos vivos; protección y preservación del medio marino; investigación científica marina; y procedimientos para la solución de controversias.

\section{COMISIÓN NACIONAL DEL LÍMITE EXTERIOR DE LA PLATAFORMA CONTINENTAL}

La Comisión Nacional del Límite Exterior de la Plataforma Continental -de ahora en adelante, COPLA- fue creada en 1997 mediante la Ley $\mathrm{N}^{\circ}$ 24.815, como el órgano estatal encargado de elaborar la presentación final del límite exterior de la plataforma continental argentina, de conformidad con la CONVEMAR y la Ley Nº 23.968 de Espacios Marítimos.

COPLA es una comisión interministerial, presidida por el Ministerio de Relaciones Exterior y Culto, e integrada por un miembro del Servicio de Hidrografía Naval y un miembro del 
Ministerio de Economía y Finanzas Públicas. Los miembros están asistidos por un Coordinador General.

La Comisión cuenta con profesionales propios de las distintas disciplinas involucradas: geodestas, hidrógrafos, geólogos, geofísicos, cartógrafos, oceanógrafos, expertos en sistemas de información geográfica, abogados y expertos en derecho internacional. Además, cuenta con la colaboración de numerosos organismos públicos y universidades relacionados con la temática.

\section{COMISIÓN DE LÍMITES DE LA PLATAFORMA CONTINENTAL}

La Comisión de Límites de la Plataforma Continental -de ahora en adelante, CLPC- fue creada en 1982 y está compuesta por 21 expertos encargados de examinar los datos y toda la información presentada por los estados ribereños sobre el límite exterior de la plataforma continental. Las reuniones de la CLPC tienen lugar una vez al año durante un mes en la sede de Naciones Unidas en Nueva York. Luego del análisis, la CLPC puede formular recomendaciones para ajustar la presentación a los criterios establecidos por la Convemar. Luego de que el estado ribereño cumpla con las recomendaciones, si las hubiera, el límite exterior de su plataforma continental así presentado es obligatorio para los países miembros. La CLPC no considera ni emite recomendaciones sobre áreas disputadas.

\section{PRESENTACIÓN ARGENTINA ${ }^{4}$}

El 21 de abril de 2009 la República Argentina presentó el límite exterior de la plataforma continental argentina ante CLPC. Los puntos más relevantes fueron expuestos oralmente por una delegación de COPLA y funcionarios de la Cancillería ante la CLPC el 26 de agosto de 2009.

En agosto de 2012, durante el $30^{\circ}$ Período de Sesiones de la CLPC, se formó la Subcomisión encargada de considerar la presentación argentina, comenzando así el proceso de análisis del límite exterior de la plataforma continental ya presentado.

La Argentina ha realizado un profundo y acabado trabajo científico y técnico que le permitió fijar el límite exterior de su plataforma continental, el cual brinda certeza sobre la extensión geográfica de nuestros derechos de soberanía sobre los recursos del lecho y subsuelo en más de 1.782.00o km2 de plataforma continental argentina más allá de las 200 millas marinas, que se suman a los aproximadamente 4.799.00o km2 comprendidos entre las líneas de base y las 200 millas marinas. 
El límite exterior de la plataforma continental de todo el territorio continental, islas del Atlántico Sur y Sector Antártico Argentino está formado por 6.336 puntos de coordenadas geográficas en WGS84. ${ }^{5}$

El límite presentado cumple con las disposiciones de la CONVEMAR y de las Directrices Científicas y Técnicas de la CLPC, y en su elaboración se procuró la utilización de todos los recursos disponibles para que el trazado de ese límite sea el más beneficioso para el país de acuerdo con los criterios y restricciones previstos.

Este es un ejemplo de una política de Estado en donde se ha trabajado con identidad de objetivos, profesionalismo y seriedad durante casi 20 años con el objetivo de reafirmar nuestra presencia, preservar nuestros recursos y reafirmar nuestros derechos soberanos en una zona política, económica y estratégicamente tan importante como el Atlántico Sur.

Durante la etapa de la presentación argentina se mantuvieron nueve rondas de sesiones de trabajo: agosto de 2012, febrero/marzo de 2013, julio/agosto de 2013, octubre/ noviembre de 2013, enero/febrero de 2014, agosto de 2014, noviembre de 2014, febrero de 2015 y agosto de 2015. Durante la sesión de agosto de 2015, la Subcomisión finalizó el análisis de la Presentación argentina, dando comienzo a la etapa de consideración por parte del Plenario de la CLPC. El 27 de agosto la Argentina realizó, de conformidad con el Reglamento de la CLPC, su presentación oral frente al Plenario.

\section{ACTUALIDAD 6}

A marzo de 2016 la Argentina había incorporado 1.700.0oo kilómetros cuadrados a su plataforma continental, es decir un 35\% más de superficie. Sin embargo, habían quedado pendientes dos puntos más septentrionales del límite exterior de la plataforma continental denominada RA-01 y RA-02 en los que no se alcanzó consenso sobre el rol que juega la Transferencia del Salado en la distorsión del margen en esa zona.

La COPLA trabajó intensamente entre marzo y octubre de 2016, obteniendo nuevos datos y realizando un reprocesamiento que permitió, sin ceder en los fundamentos científicos que contiene la presentación original, utilizar otros puntos del pie del talud para determinar los dos puntos del límite exterior pendientes. Con estos nuevos datos, el 30 de octubre de 2016 la Argentina realizó una Presentación Parcial revisada ante la CLPC.

El 14 de febrero de 2017 la Argentina realizó la exposición oral de dicha Presentación ante el Plenario de la CLPC en su $43^{\circ}$ Sesión. Luego de esta exposición, la CLPC decidió considerar

\footnotetext{
${ }^{5}$ El WGS84 (World Geodetic System 1984) es un sistema de coordenadas geográficas mundial que permite localizar cualquier punto de la Tierra (sin necesitar otro de referencia) por medio de tres unidades dadas. WGS84 son las siglas en inglés de World Geodetic System 84 (que significa Sistema Geodésico Mundial 1984).
}

${ }^{6}$ llustraciones en ANEXO 1.2. 
la Presentación Parcial Revisada durante este período de sesiones y el 14 de marzo se realizó la presentación final ante el plenario de la CLPC.

En esa misma sesión, el 17 de marzo de 2017, la CLPC adoptó las recomendaciones sobre esos dos puntos. Nuevamente la Argentina obtuvo recomendaciones positivas de parte de la Comisión de Límites de la Plataforma Continental en los dos puntos que habían quedado pendientes en la parte norte del límite. Con esto la Argentina logro incorporar 1.633 kilómetros cuadrados a su territorio.

Queda de esta manera finalizado el análisis de todos los puntos del límite exterior de la plataforma continental argentina a excepción de aquellas zonas sujetas a una disputa de soberanía con el Reino Unido, así como el sector que está regido por el Tratado Antártico. $\mathrm{Al}$ no pronunciarse sobre la zona circundante a Islas Malvinas, Islas Georgias del Sur e Islas Sándwich del Sur, la Comisión reconoció la existencia de una disputa de soberanía entre la Argentina y el Reino Unido.

\section{PROYECTO "PAMPA AZUL"}

\section{ORIGEN}

En el año 2014 se creó el "Centro de investigación y transferencia del Golfo San Jorge” formado inicialmente por la Universidad Nacional de la Patagonia San Juan Bosco y el CONICET, y que luego se amplió a la Universidad Nacional de la Patagonia Austral y organismos de Ciencia y Técnica de Chubut y Santa Cruz para más tarde sumar más universidades y cerca de siete ministerios. Son cerca de 30 investigadores los que participan y trabajan en el proyecto en el que se realizan tomas de agua, se hacen perfiles y datos oceanográficos, captura de plancton, fitoplancton y relevamientos para el ministerio de Defensa de la Nación.

Con el Proyecto Pampa Azul, se busca reivindicar la soberanía argentina en la plataforma marítima. La participación de la UNPSJB es fundamental para el programa y para la Universidad desde el punto de vista de la generación e incorporación de conocimiento y la formación de nuevos investigadores. Además de ser una iniciativa estratégica para estudiar los recursos del mar de manera integral. El mar aporta 1,5 del PBI y a partir de estos estudios se pretende llevar este aporte hasta el 15 por ciento, para eso se necesita conocer los recursos del mar con el aporte de la ciencia con estudios de mediano y largo plazo. El nombre Pampa Azul surge desde el punto de comparación con la Pampa húmeda, para pensar un ambiente marítimo tan o más productivo que la misma. 


\section{PROYECTO}

El mismo se trata de una iniciativa del Estado argentino en el cual intervienen diversas áreas.

Está coordinada desde el Ministerio de Ciencia Tecnología e Innovación Productiva a través del Gabinete Científico Tecnológico (GACTEC). Está integrada por los ministerios nacionales de Relaciones Exteriores y Culto; de Agroindustria; de Turismo; de Defensa; de Seguridad; y de Ambiente y Desarrollo Sustentable.

Cabe destacar, asimismo, el importante rol del Consejo Nacional de Investigaciones Científicas y Técnicas (CONICET), involucrado con la iniciativa a través de sus investigadores, sus centros y buques de investigación. También abarca a la Dirección Nacional del Antártico (DNA); la Comisión Nacional de Actividades Espaciales (CONAE); el Instituto Nacional de Investigación y Desarrollo Pesquero (INIDEP); el Servicio de Hidrografía Naval (SHN); el Centro Austral de Investigaciones Científicas (CADIC); el Centro Nacional Patagónico (CENPAT); la Prefectura Naval Argentina; el Instituto Argentino de Oceanografía (IADO-CONICET/UNS); el Instituto Investigaciones Marinas y Costeras (IIMyC-CONICET); el Centro de Investigaciones del Mar y la Atmósfera (CIMA-CONICET/UBA); el Instituto de Biología Marina y Pesquera Almirante Storni (UNCO); la Universidad Nacional del Comahue; la Universidad Nacional de la Patagonia San Juan Bosco; la Universidad Nacional de Tierra del Fuego, Antártida e Islas del Atlántico Sur; la Universidad Nacional de la Patagonia Austral; la Universidad Nacional de Mar del Plata; la Universidad Nacional del Sur; la Universidad Nacional de La Plata; y la Universidad de Buenos Aires.

\section{OBJETIVOS}

Dentro de los objetivos expuestos en Pampa Azul se encuentran:

- Contribuir a profundizar el conocimiento científico como fundamento de las políticas de preservación y manejo sustentable de los recursos marinos;

- Promover innovaciones tecnológicas aplicables al fortalecimiento de las industrias vinculadas al mar y al desarrollo económico de las regiones marítimas argentinas.

- Fortalecer la conciencia del patrimonio marítimo y el uso responsable de sus recursos dentro de la sociedad argentina;

- Respaldar con información y presencia científica la soberanía de nuestro país en el área del Atlántico Sur. 


\section{ÁREAS SELECCIONADAS}

Se han seleccionado cinco áreas geográficas prioritarias sobre la base de sus características oceanográficas, la importancia de sus ecosistemas y el impacto potencial de las actividades humanas:

- Banco Burdwood/Área protegida Namuncurá

- Frente del Talud Continental/Agujero Azul

- Golfo San Jorge

- Islas Subantárticas

- Sistema fluvio-marino del Río de la Plata.

\section{RECURSOS}

\section{IMPACTO ECONÓMICO}

El impacto económico no es menor. La zona adyacente a las islas Malvinas es muy rica tanto en cuanto a la pesca como a los hidrocarburos. A la Argentina ya le pertenecen todos los recursos en la zona de o a 200 millas náuticas con excepción de la zona que la Convención le adjudique a las islas Malvinas. A éstas les corresponde un territorio circular con un radio de 200 millas náuticas alrededor de las mismas. Estos recursos incluyen, por ejemplo, la pesca y la explotación de yacimientos de petróleo y gas natural. La realización de ese potencial solo sería posible si Argentina dispone del capital y de la tecnología necesaria.

En cuanto a los recursos en sí, Argentina extiende el derecho soberano sobre el fondo del mar, donde se incluye la explotación hidrocarburífera (petróleo y gas) y mineral (hierro, zinc y otros de uso industrial estratégico, como nódulos polimetálicos de manganeso, costras de cobalto o sulfuros), los recursos pesqueros o ictícolas que están apoyados sobre el fondo submarino, y las especies sedentarias -tales como la langosta, los mejillones y las vieiras -.

Estos recursos naturales adicionan no solo potencialidad económica en término de las materias primas tan requeridas en el mundo -sobre todo en las industrias de la biotecnología, la farmacéutica, y la electrónica -, sino que contribuyen a la seguridad energética y alimentaria; lineamiento central en las políticas económicas estatales actuales y a futuro. 


\section{PESCA}

El Mar Argentino es uno de los últimos grandes ecosistemas marinos que se mantiene relativamente en buen estado de conservación. En los espacios marítimos argentinos habitan más de 600 especies de vertebrados: unos 420 peces óseos, 112 peces cartilaginosos, 82 aves marinas, 55 mamíferos marinos y cuatro tortugas.

Sin embargo, la ausencia de un plan de manejo responsable, de un esquema claro y eficaz de participación, junto a las fallas en los controles, son algunas de las variables que ponen en peligro el futuro de la pesca.

La aprobación, por parte de la ONU, de la presentación argentina sobre el límite exterior de la plataforma continental ratifica el derecho de soberanía sobre los recursos del lecho y subsuelo en más de $\mathbf{1 . 7 8 2 . 0 0 0} \mathrm{km} 2$ de plataforma continental argentina, lo que implica un aumento del $35 \%$, pero, no impacta de modo directo sobre la columna de agua, por lo cual, no tiene influencia sobre la mayoría de los recursos pesqueros importantes para la región. Por ello, es necesario lograr acuerdos con Naciones Unidas para el manejo del Alta Mar, más allá del límite de la zona económica exclusiva, para abordar la pesca ilegal.

Para esto, Vida Silvestre ${ }^{7}$ propone la implementación efectiva del Programa Nacional de Investigación e Innovación Productiva en Espacios Marítimos Argentinos -PROMAR ${ }^{8}$, una iniciativa estratégica de investigaciones científicas en el Mar Argentino (antes conocida como "Pampa Azul"), que incluye actividades de exploración y conservación; de innovación tecnológica para los sectores productivos vinculados al mar; y de divulgación científica dirigida al público en general. Sus objetivos principales son:

-Desarrollar el Proceso de Planificación Marina Espacial: herramienta fundamental que facilita el ordenamiento de los espacios marinos y el balance entre las demandas del desarrollo con la necesidad de proteger los ecosistemas marinos, logrando beneficios ambientales, sociales y económicos. También brinda elementos para mejorar la gestión de los gobiernos en los espacios marinos, a través de un conocimiento integrado sobre la biodiversidad, los recursos naturales y servicios ambientales; una mejor capacidad de coordinación entre sectores, instituciones y jurisdicciones; y una anticipación de los potenciales conflictos en los usos; entre otros.

-Implementar el Manejo Basado en el Ecosistema en las pesquerías nacionales: como un enfoque integrado, que contemple todos los componentes del ecosistema, y que busque compatibilizar las necesidades sociales y económicas de las comunidades, con la conservación de ecosistemas.

${ }_{7}^{7}$ La Fundación Vida Silvestre Argentina es una organización no gubernamental, de bien público y sin fines de lucro, creada en 1977. Su misión es proponer e implementar soluciones para conservar la naturaleza, promover el uso sustentable de los recursos naturales y una conducta responsable en un contexto de cambio climático.

${ }^{8}$ Ley 27167 / 2015. 
Las Naciones Unidas han reconocido nuestros derechos sobre los territorios marítimos. Pero depende de la Argentina desarrollar un modelo pesquero integrado que garantice la riqueza de nuestros mares.

\section{HIDROCARBUROS}

\section{ÁREA OFFSHORE 9}

$\mathrm{El}$ área offshore de nuestro país alberga algunas de las cuencas que tienen bajo nivel de madurez exploratoria. Sin embargo, esta no es la característica más generalizada y, por ende, no puede adjudicarse ese estado a toda la región marina.

Se han explorado cuencas como la de Rawson y la de San Julián, o sectores de plataforma interna de Salado y Colorado.

Las áreas de plataforma externa de las cuencas de Salado y Colorado, en especial esta última, junto con la Cuenca Argentina, ameritan y necesitan exploración adicional. El potencial de la Cuenca del Golfo está claramente relacionado con el precio del petróleo. Por el momento, allí existen solamente recursos. La Cuenca Austral es la más madura desde el punto de vista exploratorio y quizás pueda agregar reservas adicionales a las ya existentes, pero en volúmenes limitados.

La región de la Cuenca de Malvinas bajo jurisdicción argentina parece restringir sus posibilidades hidrocarburíferas hacia su extremo sur, se trata, sin dudas, de una región que justifica esfuerzos adicionales. Respecto del potencial petrolero de las cuencas en las adyacencias de las Islas Malvinas, aún sigue sin definirse. Es indudable que, al finalizar la presente campaña exploratoria en Malvinas Norte, se tendrá una visión mucho más ajustada del potencial en esa cuenca, donde aparentemente se encuentran las mejores posibilidades de realizar algún posible descubrimiento.

Malvinas Oriental es una frontera de altísimo riesgo, y es costosa. Y, para el sector de la Cuenca de Malvinas bajo control británico, se aplican los mismos comentarios que para el sector bajo jurisdicción argentina. Sin lugar a duda, el acceso de nuestro país a posibles reservas en estas zonas implica aspectos políticos, sobre todo, más que técnicos.

Otro aspecto importante para tener en cuenta es que, dado el nivel de inversiones necesarias y el ya mencionado nivel de riesgo geológico, el avance de la exploración en nuestros mares parece requerir de la participación de compañías de envergadura que tengan los suficientes respaldos financieros para emprender esta actividad. Las condiciones para explorar en la plataforma deberán ser, entonces, lo suficientemente explícitas y permanentes como para atraer esas inversiones. Además, debe considerarse que, de existir reservas importantes, estas no estarían disponibles en volúmenes importantes hasta dentro de una década suponiendo 
que empezáramos hoy mismo. Por el contrario, y desde un punto de vista estratégico, la migración de nuestra matriz energética hacia combustibles no fósiles parece ser obligatoria e irreversible; este proceso debería implementarse de inmediato si no queremos quedar inmersos en una gravísima situación de insuficiencia energética, dependientes de la importación masiva de petróleo y de gas para abastecer nuestras necesidades energéticas futuras. ${ }^{10}$

\section{ACTUALIDAD}

El Ministerio de Energía y Minería otorgó a la petrolera YPF en enero de 2017 a través de la resolución $13^{11}$ un permiso de reconocimiento superficial por un plazo de 24 meses sobre un área ubicada en el sector norte del margen continental argentino mas allá de las 12 millas marinas. El área bajo estudio tiene unos 360.00o kilómetros cuadrados que van desde el límite con Uruguay hasta los $45^{\circ}$ de latitud (norte de Comodoro Rivadavia), donde las profundidades del agua van desde los 500 hasta los 3.500 metros.

A partir de esto la empresa argentina comenzaría a trabajar en el talud continental offshore argentino junto a la estatal noruega Statoil (esta última con gran experiencia en exploración en mar adentro)

A su vez, en forma simultánea se otorgó un permiso similar a la proveedora noruega de servicios sísmicos Spectrum Asa, en un área de 35.00o kilómetros, también ubicada frente a la costa de la provincia de Buenos Aires.

Los estudios que efectuarán ambas empresas resultarían de importancia para las futuras exploraciones en busca de hidrocarburos en las cuencas off shore de la República Argentina.

Los permisos contemplan la recolección de datos a través de sísmica en dos dimensiones (2D) -que consiste en el envío y recepción de ondas sonoras desde una embarcación- en una amplia superficie que abarca la zona del talud continental. A su vez se autoriza a efectuar estudios geológicos y geofísicos y emplear otros métodos orientados a la exploración petrolera.

Cabe destacar que el permiso otorgado no generará derechos a favor de YPF sobre ese territorio. Una vez finalizado el estudio y dentro de los noventa días siguientes al vencimiento del Permiso de Reconocimiento Superficial, YPF deberá presentar ante la Subsecretaría de Exploración y Producción de la Secretaría de Recursos Hidrocarburíferos del Ministerio de Energía y Minería de la Nación, conjuntamente con los datos primarios, un informe completo relativo a los trabajos emprendidos y actividades desarrolladas.

El ex ministro de Energía, Juan José Aranguren, resaltó que la búsqueda de hidrocarburos mar afuera no es inversión de corto plazo, sino que consta de un proceso de diez años.

${ }^{10}$ Ampliación de información en ANEXO 2.

${ }^{11}$ Publicada en el Boletín Oficial del 07-feb-2017. 
Eso es lo que se estima cuando se está haciendo exploración de alto riesgo en la plataforma continental argentina, una de las pocas que quedan en el mundo prácticamente inexplorada; por lo tanto, atrae el ánimo inversor, a pesar del riesgo que ello conlleva.

En el pasado, YPF invirtió US\$ 160 millones frente a las costas de Comodoro Rivadavia, aunque no consiguió hallazgos comerciales. Sin embargo, especialistas intuyen que en el Mar Argentino hay recursos petroleros importantes. Es por tal motivo que el Gobierno Nacional relanzó la búsqueda de petróleo y de gas en la plataforma continental argentina.

Durante 2018 se espera licitar tres cuencas que suman unos 225.000 kilómetros cuadrados. La primera ronda va a incluir una vasta región de la Plataforma Austral Norte (5.00o km2); la Cuenca Malvinas Oeste (90.00okm2) y la Cuenca Argentina Norte (130.00o km2)".

Se espera recibir las ofertas en el mes de noviembre de 2018 para adjudicar los Permisos Exploratorios a finales del mismo año.

El ex ministro destacó que la licitación será continuada en el año 2019 por una segunda Ronda, en la cual se incorporará la Cuenca Argentina Sur, que cuenta con un área aproximada de $120.000 \mathrm{~km} 2$.

Actualmente, la producción offshore está concentrada en la Cuenca Austral: en 2016 representó el 2\% de la producción nacional de petróleo y 17\% del gas.

\section{REFLEXIONES FINALES}

- Deberán llevarse a cabo en las zonas de interés exploratorio, trabajos de sísmica y perforaciones con la finalidad de hacer una evaluación económica de este sector, que hasta la actualidad se lo puede considerar de investigación incipiente.

- La costa afuera argentina es uno de los espacios más extensos y menos explorados con potencial de recursos hidrocarburíferos a nivel global, configurando una oportunidad única para la atracción de inversiones al país, y uno de los proyectos más importantes que tiene la Argentina para extender el horizonte de las reservas de gas y petróleo. Se cree que la explotación offshore registra mayores recursos que Vaca Muerta, sin embargo, se debe tener en cuenta que los proyectos de exploración conllevan un presupuesto mayor que el desarrollo no convencional.

- La exploración offshore difícilmente traiga soluciones en el corto plazo al déficit de hidrocarburos que hay en la Argentina, en especial de gas. Sin embargo, se considera que es un área en la que el Estado debe comenzar a promover la inversión privada.

- La pesca clandestina en nuestra plataforma es un grave problema que debemos afrontar. Las flotas extranjeras cuentan con medios electrónicos para detectar tanto 
el movimiento de cardúmenes como la posición de navíos argentinos, lo que demanda un mayor esfuerzo de control.

- El frente del talud es una región muy rica en producción primaria y pesquera y contiene la mayoría de los recursos vivos explotables.

- Urge recuperar el potencial de la Armada nacional para el patrullaje aéreo y marítimo permanente del océano. Por falta de recursos, la Armada Argentina no puede empeñar sus medios de superficie y aéreos en adecuada secuencia de patrullas para asegurar el cumplimiento de las leyes vigentes. Además, la flota está envejecida y con graves problemas de mantenimiento, a su vez carece de combustible suficiente para el patrullaje sistemático. Los bajos sueldos han provocado la disminución de personal, tanto de navegación como en la fuerza aeronaval.

Las 200 o 350 millas náuticas, y aún más allá, según los compromisos internacionales, exigen a la Armada esfuerzos de distancias y tiempos de permanencia en el mar con barcos adecuados y personal idóneo, con los cuales no cuenta.

En conclusión, consideramos que la extensión de la plataforma podría ser calificada como un hecho histórico, debido a las décadas de silencioso y pacífico trabajo que abarcó no solo varios gobiernos, sino también, entidades, universidades y otras organizaciones. Se agregan así 1.700.00o kilómetros cuadrados a los 4.800.000 ya reconocidos como argentinos, esto es, un aumento del $35 \%$ sobre la superficie anterior y que pasa a representar el $48 \%$ del territorio nacional.

La naturaleza nos bendijo con una de las plataformas más extensas y ricas del planeta, con lo cual, es un alentador pronóstico que debemos aprovechar para construir poder nacional, lo cual implica aprovechamiento racional de los recursos del mar, lecho y subsuelo marítimos, desarrollo de la industria naval, recuperación de la flota pesquera, flota de mar y aviación militar, desarrollo industrial y científico de la Patagonia. Es la única manera y exige tiempo, esfuerzo, coherencia y responsabilidad. 


\section{ANEXO}

\section{ANEXO 1- MAPA DE LA PLATAFORMA CONTINENTAL ARGENTINA ${ }^{12}$}

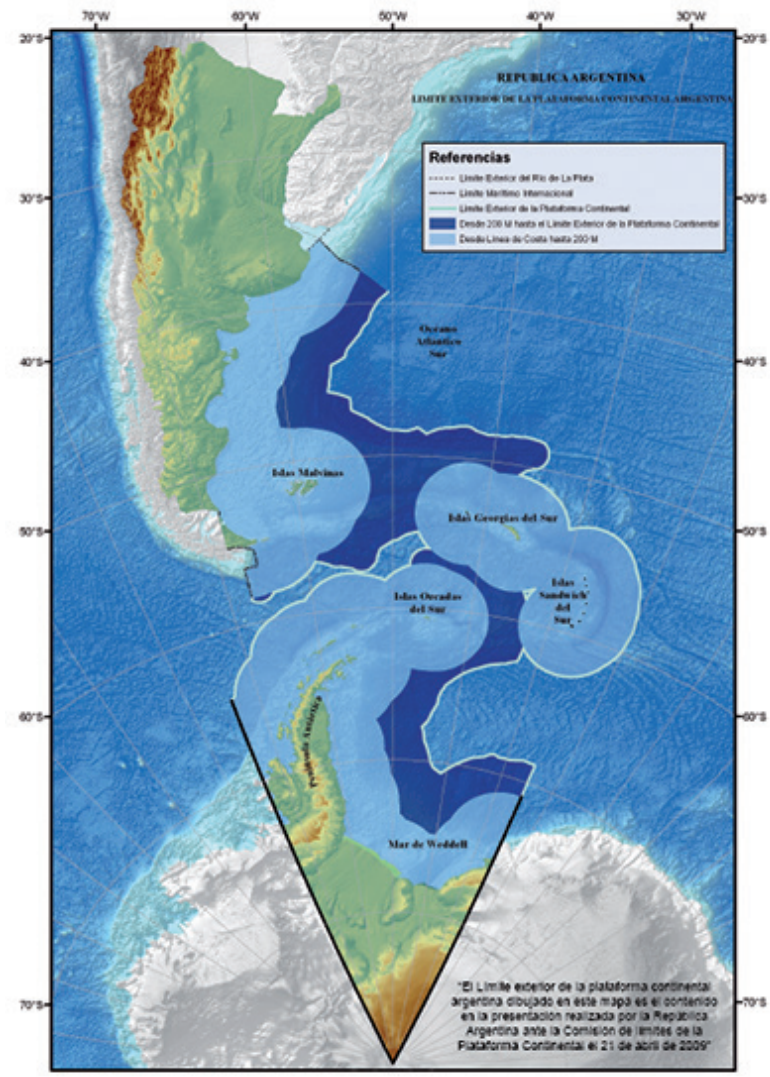

\section{ANEXO 1.1- ESQUEMA ILUSTRATIVO DE LA PLATAFORMA}

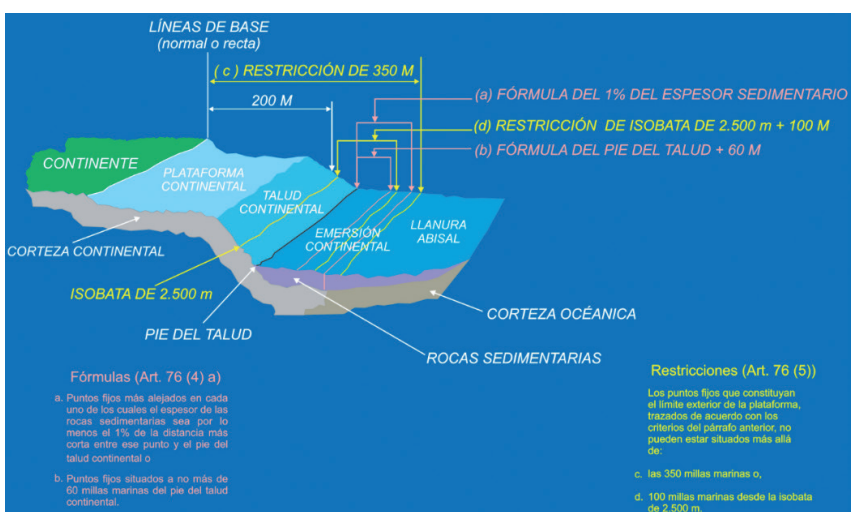

Fuente: COPLA

$\overline{{ }^{12} E l ~ l i ́ m i t e ~ e x t e r i o r ~ d e ~ l a ~ p l a t a f o r m a ~ c o n t i n e n t a l ~ a r g e n t i n a ~ d i b u j a d o ~ e n ~ e s t e ~ m a p a ~ e s ~ e l ~ c o n t e n i d o ~ e n ~ l a ~ p r e s e n t a c i o ́ n ~ r e a l i z a d a ~ p o r ~}$ la República Argentina ante la Comisión de Límites de la Plataforma Continental (CLPC) el 21 de abril de 2009. 


\section{ANEXO 1.2- ÚLTIMOS CAMBIOS EN LA PLATAFORMA APROBADOS EN MARZO DE 2017}

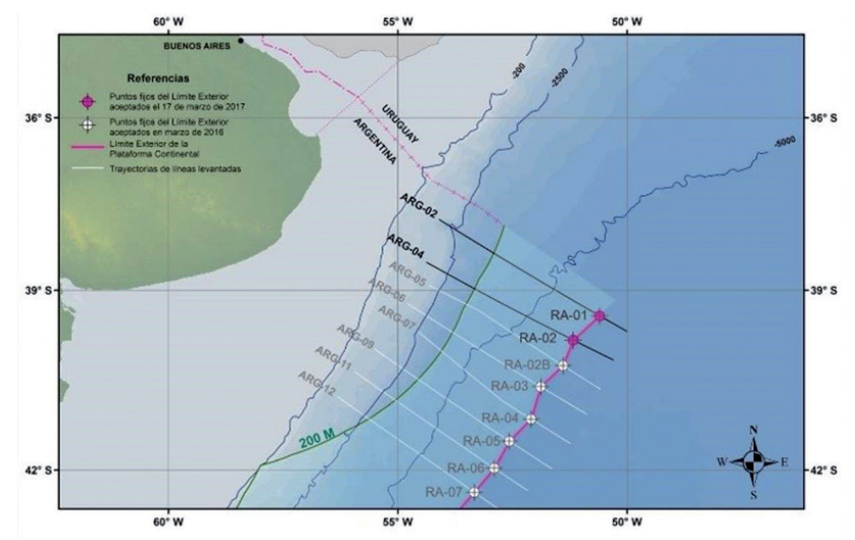

\section{ANEXO 2: CUENCAS OFFSHORE DE ARGENTINA}

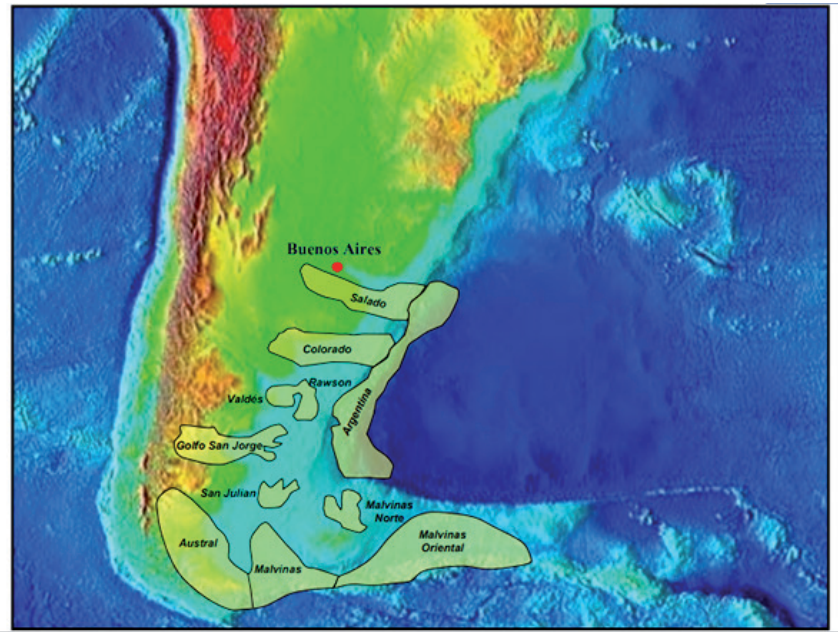

Fuente: IAPG (Instituto Argentino del Petróleo y del Gas).

\section{El prospecto Sea Lion}

El pozo fue perforado durante abril y mayo de 2010, alcanzó una profundidad final de $2744 \mathrm{~m}$. El pozo no fue ensayado, sino puesto en espera de terminación mientras la plataforma se trasladaba a perforar el prospecto Toroa en Malvinas Oriental. Se generó un comunicado de prensa, fechado el 23 de julio, en el que se mencionaba que la plataforma Ocean Guardian comenzaba a perforar el pozo Ernest; se esperaba llegar al fondo en unos treinta días y luego trasladar la plataforma para ensayar Sea Lion. En él, las arenas con 
hidrocarburos se encontraron entre los $2374 \mathrm{~m}$ (donde se ubicaría un sello regional) y los $2591 \mathrm{~m}$, lo que definía un gross interval de $217 \mathrm{~m}$. Los datos de presión permiten suponer la presencia de dos columnas de petróleo independientes y que las arenas con hidrocarburos más delgados y a mayor profundidad probablemente se encuentren muy por debajo del límite mapeado en sísmica para el Sea Lion Fan. Si bien no es posible realizar una evaluación exhaustiva del ensayo del Sea Lion por no contar con toda la información básica, del análisis de los datos publicados puede concluirse en que: La acumulación ensayada es pequeña, el reservorio parece tener muy baja transmisibilidad y por último, la presión de surgencia en boca de pozo es muy baja; debería estar en el orden de las 400-450 psi y es Petrotecnia.

Sin duda, ninguna de las características mencionadas resulta muy atractiva desde el punto de vista de la calidad de los reservorios sin embargo el valor del ensayo en el Sea Lion consiste en aportar el primer volumen de petróleo en la Cuenca de Malvinas Norte. Se trata de un hito importantísimo; sin embargo, afirmar que constituye el descubrimiento del primer yacimiento de petróleo en la cuenca es un tanto prematuro. Se requerirá de un importante esfuerzo adicional, básicamente varios pozos de delineación, para confirmar si se está en presencia de reservas de petróleo o de un recurso.

\section{El prospecto Liz}

El pozo Liz fue perforado por Desire Petroleum antes del pozo Sea Lion y fue abandonado como pozo descubridor de gas tras alcanzar la profundidad de $3667 \mathrm{~m}$. En todo caso, el pozo tuvo gas shows, y los perfiles arrojaron $17 \mathrm{~m}$ de probable gas pay, distribuidos en un intervalo arenoso de $70 \mathrm{~m}$ de espesor que se ubica entre los 2961 y los $3031 \mathrm{~m}$. Pudo también definirse que los potenciales reservorios corresponden a arenas de muy baja permeabilidad, que presentan claras muestras de sobrepresión. El pozo, según Desire Petroleum, también encontró dry gas en niveles más profundos del synrift que no pudieron ser ensayados debido a las altísimas presiones que ponían en riesgo la operación normal del pozo.

Similitudes con el Sea Lion: Se encuentran muy cerca uno del otro, y la única diferencia es que provienen de márgenes diferentes. Ambos pozos fueron perforados explorando sendas amplitudes sísmicas. El Liz es un dry hole, y el Sea Lion está en espera de terminación.

\section{El prospecto Ernest}

Está ubicado en la zona sur de la Cuenca de Malvinas Norte, donde, hasta el momento, no hay signos de roca generadora. La probada roca madre cretácica parece estar ausente aquí en ocasiones por falta de sedimentación y en otras por estar fuera de la ventana de generación de petróleo. Tampoco hay signos de potencial roca madre de edad jurásica en este depocentro. Ernest es un interesante prospecto, ya que estará investigando una situación totalmente novedosa. Es de altísimo riesgo, ya que, la posibilidad de éxito está en el orden 
del 12\% y, de ser estéril, no debería llamar la atención. Por el contrario, de resultar en un descubrimiento, generaría un completo replanteo de los modelos exploratorios utilizados hasta ahora.

\section{El prospecto Rachel}

La decisión de perforar este prospecto fue tomada luego de conocerse los resultados del pozo Sea Lion. El play concept es similar, aunque, hay que tener en cuenta algunas características que pueden aumentar el riesgo de este prospecto. La posibilidad de éxito según Desire Petroleum para el Rachel es de 15\%. Tal como lo mencionan distintos informes de la operadora, si bien este prospecto es análogo al Sea Lion, aquí no se cuenta con el relevamiento total de sísmica en $3 \mathrm{D}$, ya que no cubre el sector norte del prospecto, que sólo tiene 2D. El pozo más cercano se encuentra a unos $20 \mathrm{~km}$. Otro dato para tener en cuenta es que, aunque el prospecto fue definido por anomalías en la sísmica, no se han comprobado anomalías AVO. En conclusión, Rachel es un prospecto de mayor riesgo que el Liz y el Sea Lion, y los resultados del pozo serán muy importantes para ponderar el potencial de la subcuenca septentrional de Malvinas Norte. El 15 de octubre de 2010, Desire Petroleum anunció que el pozo 14/15- 1 Rachel alcanzó la profundidad final de $2877 \mathrm{~m}$. También se demostró como dato positivo la presencia de $81 \mathrm{~m}$ de rocas que tenían buenas características de reservorio y porosidades promedio del $23 \%$.

\section{CONCLUSIONES GENERALES}

- Gran parte de estas cuencas se cubrieron con sísmica en 2D, y la sísmica 3D fue utilizada para interpretar las áreas que presentaban mayor interés prospectivo.

- El Sea Lion constituye la primera prueba concreta de una posible trampa de hidrocarburos en Malvinas Norte. Su magnitud es absolutamente desconocida, toda vez que el pozo no se ensayó.

- En la Cuenca de Malvinas Oriental, el nivel de exploración es mucho más incipiente, es decir, está en estado inicial. El resultado del pozo perforado no es suficiente para condenar el área ni mucho menos. Es evidente que, en este sector, la inversión de riesgo requerida puede ser de mayor envergadura, puesto que gran parte de la cuenca está construida en profundidades de agua más allá de los 1000 metros. 


\section{REFERENCIAS BIBLIOGRÁFICAS}

Fisiografía de la Plataforma Continental

Gerardo Parker, Roberto A. Violante y Marcelo C. Paterlini.

Cuaternario y Dinámica Costera

Jorge O. Codignotto.

Estructura de la Plataforma Continental

Carlos M. Urien Y Juvenal J. Zambrano.

Palinoestratigrafía de la Plataforma Continental

Sergio Archangelsky.

Microfósiles y Nanofósiles Calcáreos de la Plataforma Continental

Norberto Malumián y Carolina Nañez.

Cuenca del Salado

Guillermo F. Tavella y Christopher G. Wright.

Flanco Norte de la Cuenca del Colorado

Rosana del C. Juan, Jan de Pager, Jack Russell e Irene Gebhard.

Cuenca del Colorado

Bob Fryklund, Alex Marshall y Joe Stevens.

Cuencas de Rawson y Península de Valdés

Raúl V. Marinelli y Humberto J. Franzin.

Cuenca Costa Afuera del Golfo San Jorge

Jorge E. Balde y Vicente A. Nevistic.

Cuenca de San Julián

Antonio M. F. Figueiredo, Aloisio Pellon de Miranda, Rudy F. Ferreira y Pedro V. Zalan.

Geología de las Islas Malvinas

Francisco E. Nullo, Luis Dimitri y María E. Kusiak. 
Plateau de las Malvinas

Kevin T. Biddle, Parke D. Snavly III y Miguel A. Uliana.

Cuenca de Malvinas Norte

James G. Ross, Hohn Pinchin, Dean G. Griffin, Menno G. Dinkelman, Mateo A. Turic y Vicente A. Nevistic.

Cuenca de Malvinas

José S. Galeazzi.

Cuenca de Malvinas Oriental

Pedro R. Kress, Humberto J. Franzin y Raúl V. Marinelli.

Cuenca Austral Marina

Juan A. Robbiano, Hugo Arbe y Alfredo Bangui.

Cuencas del Talud Continental

Raúl V. Marinelli, Gustavo A. Rebay y Humberto J. Franzin.

Geología de las Islas Georgias del Sur

Víctor A. Ramos.

Geología de las Islas Sándwich del Sur

Víctor A. Ramos.

Evolución Tectónica de la Plataforma Continental

Víctor A. Ramos.

Geología y Recursos Naturales de la Plataforma Continental

Mateo A. Turic, Vicente A. Nevistic y Gustavo A. Rebay.

\section{OTRAS FUENTES INFORMATIVAS}

- http://www.telam.com.ar/notas/201711/221160-argentina-espera-hallazgos-de-petroleo-ygas-en-areas-de-la-plataforma-continental-que-licitara-en-2018.html 
- https://www.infobae.com/economia/2018/03/13/empresas-internacionales-se-interesanen-la-exploracion-offshore-en-la-argentina/

• https://ar.reuters.com/article/topNews/idARL2N1MG1Y1

- http://losandes.com.ar/article/view?slug=argentina-permitira-la-busqueda-de-petroleo-enel-mar

• http://econojournal.com.ar/2017/o2/ypf-relanza-la-exploracion-de-la-plataforma-continental/

- https://www.lanacion.com.ar/1981740-reactivan-la-busqueda-de-petroleo-y-gas-en-el-marargentino

• http://econojournal.com.ar/2018/03/tierra-del-fuego-lanza-una-licitacion-de-dos-areaspetroleras/

- https://www.cronista.com/negocios/Enap-adquirio-nueva-area-para-producir-hidrocarburos-en-el-mar-20180125-0018.html

- http://www.ambito.com/90660o-aranguren-anuncio-exploracion-ioffshorei-en-la-zonadonde-se-perdio-el-submarino-ara-san-juan

• https://www.elsol.com.ar/los-cuatro-objetivos-de-la-millonaria-licitacion-petrolera

- http://www.pampazul.gob.ar

\section{GLOSARIO}

AVO:

Amplitud Versus Offset, técnica utilizada como indicador de posible existencia de hidrocarburos mediante el método de comparar la variación de las amplitudes de las respuestas sísmicas en función de la distancia fuente-receptor; herramienta que permite disminuir la incertidumbre y mitigar el riesgo.

Depocentro: Porción de la cuenca que acumula un espesor importante de sedimentos o de depósitos.

Dry Hole: (pozo seco). Pozo que no ha encontrado reservas de hidrocarburos económicamente exportables y que con frecuencia se inunda con agua subterránea. 
Gross Interval: Se refiere a la sección (intervalo) de reservorio en su totalidad, dentro del cual se encuentra el intervalo neto (net) que es portador de hidrocarburo.

Gas Shows: $\quad$ Manifestaciones de gas durante la perforación.

Gas Pay: $\quad$ Espesor útil saturado de gas.

Onshore: $\quad$ Es la actividad petrolera que se realiza en tierra.

Offshore: $\quad$ Se refiere a las actividades petroleras que se realizan en la plataforma continental y en aguas internacionales.

Prospecto: $\quad$ Un destino de exploración

Play Concept: Un play es una familia de yacimientos y/o prospectos los cuales tienen en común, la misma roca almacén, roca sello, y la misma historia de generación de hidrocarburos, migración y de carga.

Sísmica 2d: $\quad$ Permite obtener imágenes en dos dimensiones: las capas de la tierra, sus formas y sus estructuras.

Sísmica 3d: Genera gráficos tridimensionales que permiten mayor detalle para verificar la presencia o no de hidrocarburos.

Synrift: $\quad$ Conjunto de rocas sedimentarias o volcánicas que se depositan durante el proceso de subsidencia tectónica en un rift: depósito contemporáneo producto de la etapa o fase de rifting.

\section{COMO CITAR ESTA COMUNICACIÓN}

SPINELLI, Micaela A., TOMASINI, Valeria y DENTICE, Enrique. La economía del mar. Los recursos marinos de la plataforma continental ampliada. Revista de la Facultad de Ciencias Económicas - UNNE, Argentina. Volumen 21 Núm. 2, julio-diciembre 2108, ISSN 1668 - 6365. Págs. 147 - 171. DOI: http://dx.doi.org/10.30972/rfce.0213732

\section{CURRICULUM VITAE}

\section{Micaela A. Spinelli}

Licenciada en Economía. Desde el año 2017 me desempeño como investigadora del CIME (Centro de Investigación y Medición Económica) de la UNSAM (Universidad Nacional de San Martin) generando informes de panoramas económicos y financieros. A su vez dentro del mismo he desarrollado una estancia corta de investigación en IELAT (Instituto Universitario de Investigación en Estudios Latinoamericanos) en la Universidad de Alcalá de Henares (Ma- 
drid), completando así mi tesina de grado de la Licenciatura en Economía. Anteriormente me he desempeñado en el sector privado en los rubros de joyería, consultoría y cosmética de empresas prestigiosas tales como Hstern (Brasil), Accenture (US/UK) y AVON (Brasil, Chile, Uruguay) para el rubro de contabilidad, finanzas y supply chain.

mspinelli@unsam.edu.ar

\section{Valeria Tomasini}

Becaria Senior en el programa de investigación del CIME (Centro de Investigación y Medición Económica) de la UNSAM desempeñándome como asistente en investigaciones empíricas de la realidad económica, a partir del año 2008 ya incorporándome como Lic. en Administración y Gestión Empresarial comencé mi tarea como investigadora-docente participando en diversos proyectos e informes y publicaciones de carácter macroeconómico. A partir del año 2015, comencé con la coordinación de los Estudios de Impacto Socioeconómico y Ambiental para la instalación de Grandes Superficies Comerciales bajo la Ley Provincial 12.573, y la coordinación del programa de Universidades para Adultos mayores Integrados del INSSJP "UPAMI". Mi labor docente la comencé como ayudante en el año 2005 desarrollándome en las aéreas de Administración y Economía.

valeria.tomasini@unsam.edu.ar

\section{Enrique Dentice}

Coordinador del CIME (Centro de Investigación y Medición Económica de la EEyN-UNSAM), generando informes de carácter macroeconómico, proyecciones de variables económicas, tanto para las publicaciones propias de la universidad, presentación en congresos internacionales, regionales y nacionales, como también a nivel empresario. Participación activa en el relevamiento de expectativas de mercado del BCRA. Complementado por una labor docente, que resulta ser la receptora de los estudios realizados. Anteriormente me desempeñé en el sector privado en puestos relevantes en el sector financiero de empresas extranjeras. Así mismo he llevado adelante asesoramientos al estado nacional en materia de deuda externa como en conjunto con el instituto universitario de seguridad marítima en el tema de hidrovía.

enrique.dentice@unsam.edu.ar 